\title{
PERCEPCIÓN DE CARGA DE LA ENFERMEDAD EN LA PERSONA CON ENFERMEDAD CRÓNICA
}

\author{
PERCEPTION OF COST OF ILLNESS AMONG CHRONICALLY ILL \\ PEOPLE
}

\section{PERCEPÇÃO DE CARGA DA DOENÇA NA PESSOA COM DOENÇA CRÔNICA}

\author{
Angie Alarcón Rodríguez* \\ Sonia Carreño Moreno** \\ Mauricio Arias Rojas ${ }^{* * *}$
}

\begin{abstract}
RESUMEN
Objetivo: Describir el perfil y la Percepción de carga de la enfermedad en personas con enfermedad crónica e identificar correlaciones entre la Percepción de carga reportada y las variables sociodemográficas y de cuidado de la persona. Material y Método: Estudio cuantitativo, descriptivo correlacional de corte transversal realizado con una muestra de 240 personas con enfermedad crónica en tres instituciones de salud. Se midió la Percepción de carga de la enfermedad con el instrumento Carga de la Enfermedad Crónica para el Paciente GCPC-UN y las variables sociodemográficas de los participantes con una encuesta de caracterización. Para el análisis de los datos se usó estadística descriptiva y para identificar las correlaciones se usó el estadístico Rho de Spearman. Resultados: Sobre la Percepción de carga se encontró que hay mayor impacto de la enfermedad en el Disconfort físico del paciente, seguido del impacto en los Aspectos socioculturales y familiares y, finalmente, en el Sufrimiento emocional y espiritual. La Percepción de carga de la enfermedad se relacionó con la edad $(r=-, 173 ; \mathrm{p}=0,007)$, el tiempo con la enfermedad $(r=-, 182 ; \mathrm{p}=0,005)$ y el número de horas de cuidado diario $(\mathrm{r}=, 357 ; \mathrm{p}=0,000)$. Conclusión: Las personas con enfermedad crónica perciben carga por la enfermedad principalmente en lo relacionado con el Disconfort físico que esta genera. Existe una asociación entre la Percepción de carga de la enfermedad con variables sociodemográficas como la edad, el tiempo con la enfermedad y las horas de cuidado diario. En este caso los profesionales de la salud deben prestar atención a la atención integral de los pacientes con enfermedad crónica en todas sus dimensiones.
\end{abstract}

Palabras clave: Carga de la enfermedad; Enfermedad crónica; Síntomas concomitantes; Evaluación de síntomas; Pacientes.

\footnotetext{
*Enfermera, Magíster en Enfermería. Instituto Nacional de Cancerología. Bogotá. Colombia. Email: aaalarconr@unal.edu.co

**Enfermera, Doctora en Enfermería. Facultad de Enfermería, Universidad Nacional de Colombia. Bogotá. Colombia. Email: spcarrenom@unal.edu.co. Autor de correspondencia.

***Enfermero, Doctor en Enfermería (c). Facultad de Enfermería, Universidad de Antioquia. Medellín. Colombia. Email: emauricio.arias@udea.edu.co
} 


\begin{abstract}
Objective: To describe the profile and Perception of cost of illness among people with chronic disease and to identify the correlations between the reported cost and the socio-demographic and care variables of the patient. Material and Method: A quantitative, cross-sectional, descriptive, correlational study conducted with a sample of 240 people with chronic disease in three healthcare institutions. The Cost of illness perception was measured using the instrument Chronic Disease Burden for the Patient GCPC-UN and a characterization survey for the socio-demographic variables of the participants. For the data analysis, descriptive statistics were used and the Spearman's Rho to identify correlations. Results: Regarding perception of cost, it was found that there is a greater impact on the disease in the Physical discomfort of the patient, followed by the impact on Sociocultural and family aspects and, finally, on Emotional and spiritual suffering. Cost of illness was related to age $(r=-.173$; $\mathrm{p}=0.007)$, time with disease $(\mathrm{r}=-.182 ; \mathrm{p}=0.005)$ and the number of hours of daily care $(\mathrm{r}=.357 ; \mathrm{p}=0.000)$. Conclusion: People with chronic disease show Cost of illness mainly related to the Physical discomfort that it generates. There is a relationship between Cost of illness and socio-demographic variables such as age, time with disease and hours of daily care. Healthcare professionals should thus pay attention to the comprehensive care of patients with chronic disease in all its dimensions.
\end{abstract}

Keywords: Cost of illness; Chronic disease; Concomitant symptoms; Symptom assessment; Patients.

\title{
RESUMO
}

Objetivo: Descrever o perfil e a Percepção de carga da doença em pessoas com doença crônica e identificar a correlação entre os efeitos reportados e as variáveis sociodemográficas e de cuidado das pessoas. Material e Método: Estudo quantitativo, descritivo correlacional, de corte transversal realizado com uma amostra de 240 pessoas com doença crônica, em três instituiçôes de saúde. A Percepção de carga da doença foi medida com o instrumento Carga da Doença para o Paciente GCPC-UN e as variáveis sociodemográficas dos participantes com uma pesquisa de caracterizaçáo. Para analisar os dados foi utilizada a estadística descritiva e para identificar a correlação utilizou-se o estadístico de Rho de Spearman. Resultados: Em relação à Percepção de carga da doença, identificou-se que existe maior impacto na doença no Desconforto físico do paciente, seguido do impacto nos Aspectos socioculturais e familiares e, finalmente, no Sofrimento emocional e espiritual. A Percepçáo de carga da doença teve relação com idade $(r=-, 173 ; p=0,007)$, tempo com a doença $(r=-, 182 ; p=$ 0,005) e o número de horas de cuidado por dia $(r=, 357 ; \mathrm{p}=0,000)$. Conclusão: As pessoas com doença crônica apresentam Carga da doença relacionada, principalmente, com o Desconforto físico que produz. Existe uma relação entre a Percepção de carga da doença e as variáveis sociodemográficas como idade, tempo com a doença e horas de cuidado por dia. Os profissionais da saúde, neste caso, devem prestar atenção ao cuidado integral dos pacientes com doença crônica em todas suas dimensóes.

Palavras-chave: Carga da Doença; Doença crônica; Sintomas concomitantes; Avaliação de sintomas; Pacientes.

Fecha de Recepción: 14/01/2019

Fecha de Aceptación: 09/07/2019

\section{INTRODUCCIÓN}

Las enfermedades crónicas (EC) han sido calificadas como una epidemia mundial que aportará 55 millones de defunciones en 2030, deteriorando el desarrollo económico y social de todos los países del mundo. El plan de acción mundial para el control de las enfermedades crónicas se propone como meta acelerar la reducción de la carga a 2020, con una visión que pretende eximir la carga evitable de dichas enfermedades en busca de alcanzar salud y productividad, como medios para superar obstáculos para el desarrollo socioeconómico ${ }^{(1)}$. En 2016 se reportó un crecimiento rápido de 
años vividos con discapacidad, relacionado con enfermedades no transmisibles, debido al crecimiento y envejecimiento poblacional, lo que prevé un aumento en el costo y demanda de servicios socio-sanitarios ${ }^{(2)}$.

De forma tradicional, la carga ha sido medida a partir de indicadores de morbilidad, mortalidad y discapacidad ${ }^{(3,4)}$, sin embargo, en virtud de que este fenómeno no solo afecta el desarrollo económico de los países, sino también el desarrollo social, merece ser explorado desde abordajes más integrativos que permitan describir el peso que la enfermedad ejerce sobre las personas que la sufren ${ }^{(5,6)}$.

La carga de la EC para el paciente es definida por Sánchez et al. ${ }^{(7)}$, como el juicio que la persona hace sobre el sufrimiento emocional y espiritual, el disconfort físico y la alteración socio-familiar y cultural, derivados de la vivencia de la EC, en términos de frecuencia, intensidad y percepción de amenaza. Bajo esta perspectiva, en Colombia se genera carga en las personas, principalmente, debido a las enfermedades cardiovasculares y el cáncer, las cuales tienen las principales tasas de morbilidad y mortalidad con indicadores que rondan el 51\% del total de las muertes en el país. Por ejemplo, en el estudio de Carrillo et al. ${ }^{(8)}$ en 2017 , se reportó que los pacientes con cáncer gástrico reportaban una alta carga emocional evidenciada por tristeza, angustia e incertidumbre; una carga física manifestada en las molestias gastrointestinales, agotamiento y debilidad y una carga socio-familiar debido a dificultades laborales y económicas.

En este contexto, cuidar a una persona con EC exige al profesional de enfermería entender que la carga es un constructo multidimensional que puede generar mayor o menor impacto en la persona, no solo debido al tipo de enfermedad que se padezca, sino también, debido a otras condiciones que caracterizan a la persona, como su entorno, relaciones sociales, edad, nivel económico o educativo, apoyo social percibido y condiciones del sistema de salud, entre otras. De acuerdo a lo anterior, es importante que durante el abordaje de la carga de la EC, no solo se aborde lo relacionado con la carga física que genera la enfermedad, sino que se tengan en cuenta los demás aspectos que la influencian, con el fin de hacer un abordaje holístico del sujeto de cuidados. Las condiciones de carga de la enfermedad de personas que padecen afecciones crónicas de salud son distintas a las de otros tipos de poblaciones ${ }^{(7)}$, por esta razón el presente estudio tuvo como objetivo describir el perfil y la percepción de carga de enfermedad de personas con EC e identificar correlaciones entre las características sociodemográficas y del cuidado con la Percepción de carga de la enfermedad reportada.

\section{MATERIAL Y MÉTODO}

Tipo de estudio y unidad de análisis: Estudio de abordaje cuantitativo, descriptivo y correlacional de corte transversal realizado durante el ańo 2018 cuya unidad de análisis estuvo constituida por personas mayores de edad con diagnóstico de EC por un periodo mayor de seis meses y que pudieran comunicarse.

Población y muestra: El estudio se realizó en la ciudad de Bogotá, Colombia, en tres instituciones de salud: la primera, de atención a personas con diversas patologías; la segunda, de atención a personas con cáncer; y la tercera, una asociación de personas con Esclerosis Lateral Amiotrófica (ELA). Estas instituciones se escogieron debido a que en su visión institucional, el cuidado de las personas con EC era uno de sus ejes y, así mismo, en su área de atención de este tipo de pacientes eran referentes para la ciudad.

La muestra se calculó considerando una correlación bivariada con una prueba a dos colas, un coeficiente de correlación como hipótesis alternativa de 0,23 , probabilidad de error tipo 1 de 0,05 y poder del $95 \%$, dando como resultado una muestra de 239 participantes. El coeficiente de correlación se tomó de los resultados de un estudio preliminar en el que se validó el instrumento ${ }^{(7)}$.

Debido a la dificultad que tuvieron los investigadores para acceder a los registros de la población de pacientes con EC en las tres instituciones y como consecuencia, aplicar un muestreo aleatorio, se optó por un muestreo por conveniencia con aquellas personas con EC que asistían diariamente a las instituciones de salud señaladas, durante los días hábiles de trabajo y en horario de 7 a.m. a 12 p.m. Para reclutarlas se invitó a participar a 299 sujetos, 90 de los servicios de hospitalización, 172 de consulta externa y 37 de urgencia. De ellas, 59 personas no participaron debido a que reusaron participar, ya habían sido 
abordadas en otro de los servicios o no cumplían los criterios de inclusión: tener un deterioro cognitivo relacionado con la enfermedad o tratamiento y que no se pudieran comunicar o responder a las preguntas. Así, la muestra final estuvo constituida por 240 personas.

Recolección de datos: Para identificar las características de las personas con EC se utilizó la Encuesta de Caracterización del Paciente con Enfermedad Crónica (GCPC-UN-P) ${ }^{(9)}$, que tiene validez facial y de contenido para Colombia. Esta encuesta indaga aspectos relacionados con el perfil sociodemográfico de la persona como edad, nivel educativo, tiempo con la enfermedad y estado civil. También incluye el perfil PULSES (Estabilidad patológica, Utiliza miembros superiores, Locomoción, capacidad Sensorial, Eliminación, actividad Social), que valora el grado de funcionalidad que tiene la persona con EC. Puntajes menores de 6 señalan total independencia de la persona, puntajes de 6 hasta 8 indican baja dependencia, puntajes de 9 a 11 indican compromiso moderado de la funcionalidad y mayores a 12 un compromiso severo de la funcionalidad.

Para medir la percepción de carga de la enfermedad, se utilizó el instrumento Carga de la Enfermedad Crónica para el Paciente (GCPCUN), desarrollado a partir de la Teoría de Manejo del Síntoma ${ }^{(10)}$ y la Teoría de Síntomas Desagradables $^{(11)}$. El instrumento consta de 48 ítems distribuidos en tres dimensiones con una escala tipo Likert con 4 opciones de respuesta que van desde 0 a 3 puntos: 15 ítems corresponden a la dimensión Sufrimiento emocional y espiritual que puntúan de 0 a 45 puntos; 18 a Disconfort físico con una puntuación de 0 a 54; y 15 ítems a Alteraciones socio-familiares y culturales con una puntuación de 0 a 45. La medición de los ítems se hace en términos de la frecuencia, la intensidad o el grado de fuerza con que dicha condición se presenta y la percepción que tiene el paciente respecto a que si esta condición amenaza su vida. Puntuaciones elevadas indican mayor Percepción de carga para el paciente. El instrumento cuenta con pruebas de validez y confiabilidad para Colombia, reportando un Alfa de Cronbach de 0,925 ${ }^{(7)}$. En esta investigación el instrumento tuvo un valor de 0,86 .
Procedimiento: El proyecto contó con el aval ético de la Universidad Nacional de Colombia y de las tres instituciones de salud donde se recolectó la muestra. La aplicación de los formatos fue realizada por dos profesionales de enfermería, auxiliares de investigación, quienes se entrenaron previamente en los objetivos del proyecto y en la aplicación de los instrumentos. Para disminuir el sesgo por conveniencia y tener un mayor rango de captación, las personas con EC fueron invitadas a participar en las salas de espera de los servicios de consulta externa, hospitalización y urgencia de las instituciones donde asistían. Una vez estos manifestaban interés, el auxiliar de investigación verificaba la historia clínica del paciente para verificar el cumplimiento de los criterios de inclusión y ayudaba a esclarecer dudas sobre el proyecto previo a firmar el consentimiento informado. Después de ser incluidos en el estudio se programaba una cita para la aplicación de los instrumentos en un espacio de privacidad para los participantes. En promedio el tiempo estimado para la aplicación de los instrumentos fue de 30 minutos. Para evitar sesgos de información, los auxiliares de investigación se adhirieron a un manual de procedimientos de aplicación del instrumento y un protocolo de digitación de los datos. Además, se realizó un control interno para hacer control al dato, que permitió auditar el 10\% del total de las encuestas.

Análisis de los datos: Los datos recolectados fueron sistematizados en Microsoft Excel $^{\oplus}$ 2013, y analizados en el software SPSS versión 23. No se identificaron datos ausentes y para evitar sesgos en el procesamiento de los datos, el investigador que los procesó estuvo ajeno al proceso de recolección. Las variables categóricas fueron tratadas con frecuencias relativas, las variables continuas fueron tratadas mediante la construcción de intervalos de confianza del 95\%, media y desviación estándar. La variable principal, carga de la enfermedad, se midió como cuantitativa de razón.

Las correlaciones fueron realizadas entre la variable carga de la enfermedad y las variables sociodemográficas de los participantes. Para estos análisis se usó el estadístico Rho de Spearman, dado que después de realizar los análisis de normalidad no se contó con ajuste al modelo Gaussiano. Aunque el coeficiente de correlación para las pruebas se fijó en 
0,23, el sistema arrojó correlaciones positivas por debajo de este valor. Dada la necesidad de establecer asociaciones entre la carga de la enfermedad y el perfil del paciente con sus características sociodemográficas, ninguna variable se tomó como variable de confusión en el desarrollo de las correlaciones. Las variables de confusión como el deterioro cognitivo o la capacidad para comunicarse fueron controladas a través de los criterios de inclusión.

\section{RESULTADOS}

Perfil sociodemográfico y de cuidado de las personas con EC y Percepción de carga de la enfermedad: La Tabla 1 presenta las características de las 240 personas con EC que se incluyeron en el estudio. Los datos representan una muestra heterogénea de la población, que varía desde el tipo de enfermedad al nivel educativo. De forma general, la población de este estudio estuvo constituida por mujeres en un 68\%; acerca de la edad se encontró que los rangos oscilaban entre 61 y 65 años (intervalo de confianza 95\%); que en su mayoría son pacientes con un compromiso leve de funcionalidad (91,25\%), pluripatológicos $(47,50 \%)$, con un grado máximo de escolaridad de nivel primario $(47,92 \%)$, mayormente casados $(30,42)$, cuya principal ocupación es en labores del hogar $(64,17 \%)$ y un nivel socioeconómico muy bajo $(39,58 \%)$.

Respecto a las variables de cuidado representadas por el tiempo con la EC y el número de horas que requiere de cuidado se encontró que el tiempo con la enfermedad estaba entre los 105,46 y los 137,30 meses (intervalo de confianza 95\%), y el número de horas requeridas de cuidado diario entre 3,18 y 4,92 (intervalo de confianza 95\%).

Tabla 1. Características de los pacientes con EC, $n=240$.

\begin{tabular}{llrc}
\hline Variables & & Fr & $\%$ \\
\hline Sexo & Femenino & 163 & 68 \\
& Masculino & 77 & 32 \\
Edad & $18-38$ & 16 & 6,73 \\
& $39-48$ & 16 & 6,73 \\
& $49-58$ & 52 & 21,67 \\
& $59-68$ & 52 & 21,67 \\
& $69-78$ & 64 & 26,67 \\
PULSES (grado de funcionalidad) & <9-98 6 & 40 & 16,63 \\
& 6 a 8 & 0 & 0 \\
& 9 a 11 & 219 & 91,25 \\
Tipo de Enfermedad & 12 a 24 & 17 & 7,08 \\
& Cáncer & 4 & 1,64 \\
& ELA & 23 & 9,58 \\
& Hipertensión arterial & 32 & 6,25 \\
& Pluripatológico & 114 & 47,33 \\
& Artritis - Artrosis & 10 & 4,17 \\
& Diabetes & 11 & 4,58 \\
& EPOC & 7 & 2,92 \\
& Otras & 28 & 11,67 \\
\hline
\end{tabular}


Continuación Tabla 1.

\begin{tabular}{llrc}
\hline Variables & & Fr & \% \\
\hline Grado máximo de escolaridad & Analfabeta & 41 & 17,08 \\
& Primaria & 115 & 47,92 \\
& Secundaria & 45 & 18,75 \\
& Técnico & 15 & 6,25 \\
& Pregrado & 15 & 6,25 \\
& Posgrado & 9 & 3,75 \\
Estado Civil & Casado(a) & 73 & 30,42 \\
& Separado(a) & 33 & 13,75 \\
& Soltero(a) & 52 & 21,67 \\
& Unión libre & 24 & 10 \\
& Viudo(a) & 58 & 24,17 \\
Ocupación & Empleado(a) & 33 & 13,75 \\
& Estudiante & 6 & 2,5 \\
& Hogar & 154 & 64,17 \\
& Pensionado & 6 & 2,5 \\
& Trabajo independiente & 41 & 17,08 \\
& Muy Bajo & 96 & 40 \\
& Bajo & 70 & 29,6 \\
& Medio & 68 & 28,3 \\
& Alto & 6 & 2,08 \\
\hline
\end{tabular}

Respecto a la Percepción de carga de la enfermedad de la persona con EC, la Tabla 2 presenta los valores alcanzados por dimensiones y en términos de frecuencia, intensidad y amenaza. Al respecto, se encontró que la dimensión Disconfort físico, en términos de frecuencia $(\bar{X}=19,32)$, intensidad $(\bar{X}=11,91)$ y amenaza $(\bar{X}=9,78)$, obtuvo los puntajes más elevados dentro de la escala; en contraste, la dimensión Sufrimiento emocional y espiritual obtuvo los puntajes más bajos en la frecuencia $(\bar{X}=12,07)$, intensidad $(\bar{X}=5,93)$ y amenaza $(\overline{\mathrm{X}}=4,66)$.

A continuación se presentan los valores alcanzados en los ítems de cada una de las 3 dimensiones que conforman la Percepción de carga de la enfermedad, según frecuencia, intensidad y amenaza:

1. Sufrimiento emocional y espiritual: En la Tabla 3 se observa que el ítem Preocupación fue el que presentó las puntuaciones más altas $(\bar{X}=1,5)$, seguido por la Labilidad relacionada con la enfermedad $(\overline{\mathrm{X}}=1,3)$. Por el contrario, el ítem Vergüenza es el que los participantes puntuaron con valores más bajos $(\overline{\mathrm{X}}=0,3)$.

2. Disconfort físico: En la Tabla 4 encontramos que el ítem Dolor fue el síntoma que se presentó con más frecuencia $(\bar{X}=1,7)$, mayor intensidad $(\overline{\mathrm{X}}=1,1)$ y que más amenaza $(\overline{\mathrm{X}}=1,0)$ representó para la vida en los participantes. La alteración de la función motora fue el segundo síntoma con más frecuencia $(\bar{X}=1,6)$.

3. Alteraciones socio-familiares y culturales: La Tabla 5 permite observar que los ítems que generan más carga en los participantes son: Cambio en las costumbres $(\bar{X}=1,9)$, seguido por Dependencia económica $(\overline{\mathrm{X}}=1,6)$ y Alteración laboral $(\overline{\mathrm{X}}=1,4)$.

Correlación entre Características sociodemográficas y de cuidado con la Percepción de carga de la enfermedad en personas con EC: Se encontró una correlación entre una de las variables 
sociodemográficas (edad) y las dos variables de de horas de cuidado diario con el Disconfort físico cuidado (Tiempo con la enfermedad y Número de $(\mathrm{Rho}=0,410)$, seguido por la correlación entre horas de cuidado diario) con la Percepción de carga la Edad y el Sufrimiento emocional y espiritual de la enfermedad. En la Tabla 6 se observa que el $\quad($ Rho= $-0,267)$.

mayor nivel de correlación se dio entre el Número

Tabla 2. Valores de la Percepción de carga de la enfermedad distribuidos por dimensiones según frecuencia, intensidad y amenaza $(n=240 /$ IC=95\%).

\begin{tabular}{lcccccc}
\hline & \multicolumn{2}{c}{ Frecuencia } & \multicolumn{2}{c}{ Intensidad } & \multicolumn{2}{c}{ Amenaza } \\
\cline { 2 - 6 } Dimensiones & Media & SD & Media & SD & Media & SD \\
\hline Sufrimiento emocional y & 12,07 & 7,16 & 5,93 & 7,71 & 4,66 & 6,02 \\
espiritual & 19,32 & 8,29 & 11,91 & 9,55 & 9,78 & 7,12 \\
$\begin{array}{l}\text { Disconfort físico } \\
\text { Alteraciones socio familiares }\end{array}$ & 13,02 & 7,91 & 8,3 & 8,13 & 5,75 & 5,27 \\
y culturales & & & & & & \\
\hline
\end{tabular}

Tabla 3. Valores de la Percepción de carga de la enfermedad según ítems de la dimensión Sufrimiento Emocional y Espiritual ( $\mathrm{n}=240$ / IC=95\%).

\begin{tabular}{lcccccc}
\hline & \multicolumn{2}{c}{ Frecuencia } & \multicolumn{2}{c}{ Intensidad } & \multicolumn{2}{c}{ Amenaza } \\
\cline { 2 - 7 } Ítem & Media & SD & Media & SD & Media & SD \\
\hline Abandono & 0,4 & 0,7 & 0,2 & 0,6 & 0,1 & 0,6 \\
Preocupación & 1,5 & 0,8 & 0,8 & 1,0 & 0,7 & 0,9 \\
Angustia & 1,1 & 0,9 & 0,5 & 1,0 & 0,4 & 0,8 \\
Baja autoestima & 0,6 & 0,9 & 0,2 & 0,7 & 0,2 & 0,6 \\
Desconfianza & 0,4 & 0,8 & 0,2 & 0,6 & 0,2 & 0,6 \\
Desesperanza & 0,4 & 0,7 & 0,1 & 0,5 & 0,1 & 0,5 \\
Culpa & 0,6 & 0,8 & 0,3 & 0,8 & 0,2 & 0,6 \\
Incertidumbre & 0,7 & 0,9 & 0,4 & 0,9 & 0,3 & 0,7 \\
Labilidad & 1,3 & 0,9 & 0,6 & 0,9 & 0,5 & 0,9 \\
Melancolía & 1,0 & 0,8 & 0,5 & 0,9 & 0,4 & 0,8 \\
Tristeza & 1,0 & 0,9 & 0,5 & 0,9 & 0,3 & 0,7 \\
Pérdida de identidad & 1,2 & 0,9 & 0,6 & 1,1 & 0,4 & 0,7 \\
Temor & 0,7 & 0,8 & 0,3 & 0,8 & 0,2 & 0,7 \\
Vergüenza & 0,3 & 0,7 & 0,2 & 0,7 & 0,1 & 0,4 \\
Disminución capacidad & 0,9 & 0,8 & 0,5 & 0,9 & 0,5 & 0,9 \\
intelectual & & & & & & \\
\hline & & & & & & \\
\end{tabular}


Tabla 4. Valores de la Percepción de carga de la enfermedad según ítems de la dimensión Disconfort Físico $(\mathrm{n}=240 / \mathrm{IC}=95 \%)$.

\begin{tabular}{lcccccc}
\hline & \multicolumn{2}{c}{ Frecuencia } & \multicolumn{2}{c}{ Intensidad } & \multicolumn{2}{c}{ Amenaza } \\
\cline { 2 - 7 } Ítem & Media & SD & Media & SD & Media & SD \\
\hline Cansancio & 1,5 & 0,9 & 1,0 & 1,1 & 0,7 & 0,9 \\
Alteraciones cutáneas & 0,7 & 1,0 & 0,3 & 0,8 & 0,3 & 0,7 \\
Alteración en actividades diarias & 1,3 & 1,1 & 0,9 & 1,2 & 0,6 & 0,9 \\
Alteración funcionalidad motora & 1,6 & 1,0 & 1,0 & 1,1 & 0,8 & 0,9 \\
Alteración sensorial & 1,1 & 1,0 & 0,6 & 0,9 & 0,6 & 1,0 \\
Debilidad & 1,2 & 0,9 & 0,7 & 1,1 & 0,5 & 0,8 \\
Desfiguración & 0,8 & 0,9 & 0,5 & 0,9 & 0,3 & 0,7 \\
Alteraciones en la comunicación & 0,3 & 0,7 & 0,2 & 0,7 & 0,2 & 0,6 \\
Alteración en la sexualidad & 0,5 & 0,9 & 0,3 & 0,8 & 0,2 & 0,5 \\
Dolor & 1,7 & 1,0 & 1,1 & 1,1 & 1,0 & 1,0 \\
Fatiga & 1,3 & 0,9 & 0,8 & 1,2 & 0,5 & 0,8 \\
Alteración del equilibrio & 1,2 & 1,1 & 0,8 & 1,1 & 0,7 & 1,1 \\
Alteraciones del sueńo & 1,0 & 0,9 & 0,5 & 0,9 & 0,5 & 0,9 \\
Molestias gastrointestinales & 1,1 & 0,9 & 0,6 & 1,1 & 0,5 & 0,9 \\
Molestias urinarias & 0,8 & 1,0 & 0,5 & 1,0 & 0,5 & 0,9 \\
Molestias respiratorias & 1,0 & 1,1 & 0,7 & 1,1 & 0,6 & 1,1 \\
Molestias osteomusculares & 1,5 & 1,0 & 1,0 & 1,1 & 0,9 & 1,0 \\
Cambios de peso & 0,9 & 0,8 & 0,4 & 0,9 & 0,3 & 0,8 \\
\hline
\end{tabular}

Tabla 5. Valores de la Percepción de carga de la enfermedad según ítems de la dimensión Alteraciones socio-familiares $y$ culturales $(n=240 /$ IC $=95 \%)$.

\begin{tabular}{lcccccc}
\hline \multirow{2}{*}{ Ítem } & \multicolumn{2}{c}{ Frecuencia } & \multicolumn{2}{c}{ Intensidad } & \multicolumn{2}{c}{ Amenaza } \\
\cline { 2 - 6 } & Media & SD & Media & SD & Media & SD \\
\hline Aislamiento & 0,5 & 0,8 & 0,2 & 0,7 & 0,1 & 0,4 \\
Alteración dinámica familiar & 1,0 & 0,9 & 0,5 & 0,9 & 0,3 & 0,6 \\
Alteración del rol en la familia & 0,5 & 0,8 & 0,2 & 0,7 & 0,2 & 0,5 \\
Alteración laboral & 1,4 & 1,2 & 1,0 & 1,2 & 0,8 & 1,1 \\
Impacto en el cuidador & 0,8 & 0,9 & 0,5 & 1,0 & 0,2 & 0,6 \\
Dependencia económica & 1,6 & 1,2 & 1,3 & 1,3 & 1,1 & 1,2 \\
Alteración en actividades recreativas & 0,9 & 0,9 & 0,4 & 0,9 & 0,3 & 0,7 \\
Afectación en la interacción social & 0,6 & 0,9 & 0,3 & 0,8 & 0,2 & 0,6 \\
Discriminación & 0,3 & 0,7 & 0,2 & 0,6 & 0,1 & 0,5 \\
Cambio en las costumbres & 1,9 & 1,0 & 1,3 & 1,1 & 0,7 & 0,9 \\
Estigmatización & 0,3 & 0,7 & 0,2 & 0,7 & 0,1 & 0,5 \\
Limitación de la movilidad & 0,9 & 1,0 & 0,5 & 1,0 & 0,4 & 0,8 \\
Alteración del estatus social & 0,5 & 0,9 & 0,4 & 0,9 & 0,2 & 0,6 \\
Sometimiento & 0,5 & 0,8 & 0,3 & 0,8 & 0,2 & 0,5 \\
Dificultades económicas & 1,4 & 1,0 & 1,0 & 1,2 & 0,9 & 1,1 \\
\hline
\end{tabular}




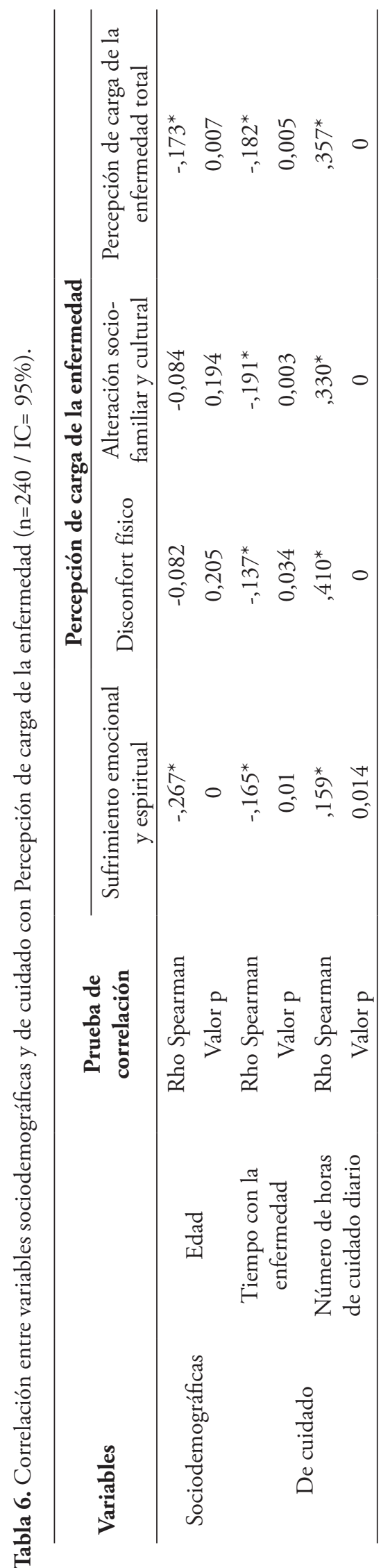

\section{DISCUSIÓN}

Sobre los niveles de Percepción de carga de la enfermedad, llamó la atención un mayor reporte de la carga física comparado con las otras dimensiones, debido, probablemente, a la dificultad para reconocer la carga no física, pues el no contar con una manifestación corporal fácilmente reconocible, dificulta la identificación del factor que produce carga. Este hallazgo es coherente con lo reportado por Palma et al. ${ }^{(12)}$, quienes, en el campo de los síntomas, encontraron que los pacientes crónicos tenían mayor dificultad en autoinformar síntomas psicológicos y sociales que cuando se trataba de síntomas físicos, sumado a una alta discordancia en la valoración de estas dimensiones entre los profesionales y los pacientes. A pesar de que este estudio no revela mayor afectación en estas áreas, la literatura ha evidenciado que la percepción de carga emocional, espiritual, social, familiar y cultural, es un problema frecuente para las personas con $\mathrm{EC}^{(13-15)}$ y de relevancia clínica para los profesionales de salud, que deben procurar el reconocimiento y el reporte de la carga de la enfermedad con el fin de brindar abordajes de atención más integradores, que vayan más allá de asistir solo la carga física y/o manejar los síntomas.

Otro hallazgo relevante es el puntaje superior de reporte de carga en términos de Frecuencia respecto de los puntajes en Intensidad y Amenaza, situación que se puede presentar por la complejidad en la configuración del significado de la experiencia de carga de la enfermedad. En efecto, en primera instancia, para la persona es más fácil identificar la ocurrencia o frecuencia de eventos que lo cargan, y el paso a evaluar un evento en términos de intensidad y amenaza, es un proceso más complejo que implica procesos cognitivos en los que interactúan factores como el estatus cognitivo, la capacidad sensorial, la exposición repetida a variados eventos, las creencias y el entorno ${ }^{(10,16)}$. Según lo anterior, es imperativo ampliar la investigación y el abordaje profesional sobre los factores contextuales en todas las dimensiones aquí señaladas, con el propósito de desarrollar intervenciones multimodales que respondan a la complejidad de la experiencia de carga de la enfermedad ${ }^{(17)}$.

En cuanto al sufrimiento emocional y espiritual, se observó una mayor expresión de carga en términos de preocupación, angustia, labilidad, 
melancolía, tristeza y pérdida de identidad. En otros estudios como el de Alpert ${ }^{(18)}$ se muestra un comportamiento similar en pacientes con falla cardiaca, donde había un alto distrés emocional caracterizado por ansiedad y baja motivación. En su estudio estos autores discuten la importancia de identificar y manejar la carga emocional en los pacientes, ya que la misma impacta en la habilidad de la persona para afrontar y manejar las actividades de la vida diaria, dentro de las que se encuentra el autocuidado y la adherencia a los tratamientos. Así mismo, para el manejo de estos síntomas de tipo emocional, la literatura recomienda identificar si estos requieren una intervención de tipo individual, por grupos de síntomas o por un síntoma centinela, que al intervenirse mejore la experiencia de los demás ${ }^{(19)}$.

La mayor carga física se presentó en términos de cansancio, alteración en la capacidad de realizar las actividades de la vida diaria, alteración de la funcionalidad motora, alteración sensorial, debilidad, dolor, fatiga, alteraciones del equilibrio y sueño, además de molestias gastrointestinales, respiratorias y osteomusculares. Dado que el $47 \%$ de los participantes se clasificaron como pacientes pluripatológico, se puede esperar que el reporte de carga en la dimensión física sea más alto y de un espectro más amplio debido a los síntomas que trae cada una de estas enfermedades. De igual manera, el reporte de síntomas como el dolor podría estar relacionado con la alta prevalencia que este tiene en poblaciones con EC, independientemente del tipo diagnóstico ${ }^{(20)}$.

Por su parte, en la dimensión Socio-familiar y cultural, la carga se expresó mayormente en las alteraciones de la dinámica familiar, afectación laboral, dificultades y dependencia económica y cambio en las costumbres. Estos resultados son explicados por otros estudios ${ }^{(3)}$ señalando que el impacto que tiene la EC en el cambio de las dinámicas familiares, sobre todo en aquellas donde las personas tenían empleos y aportaban económicamente al hogar, da lugar, en algunos casos, a un cambio de roles dentro de la familia y a un aumento de la carga económica debido a los gastos relacionados con la enfermedad.

Acorde con Dodd ${ }^{(10)}$, la experiencia del síntoma reviste una dinámica compleja por cuanto en ella confluyen el dominio de la persona, la saludenfermedad y el entorno. En este estudio y considerando que el constructo evaluado se deriva del modelo de manejo del síntoma, fue relevante describir el dominio persona, mediante el perfil de quienes padecen EC. Se observaron características de los participantes que son relevantes para describir la Percepción de carga de la enfermedad. En primer lugar, el bajo reporte de Percepción de carga de enfermedad obtenido puede deberse a que el $91,25 \%$ de los participantes tenían adecuada funcionalidad, aun cuando el $47,5 \%$ se clasificó como pluripatológico, es decir, con dos o más enfermedades crónicas primarias, situación de la que se esperaría una mayor disfuncionalidad $y$, por ende, una mayor Percepción de carga de la enfermedad ${ }^{(21)}$. Conviene, entonces, que futuras investigaciones indaguen sobre el efecto real de la condición de pluripatología en la funcionalidad y en la carga de enfermedad.

Así mismo, variables del dominio persona se correlacionaron con la carga. Se observó que la edad tuvo una correlación negativa, débil y significativa con la carga total y la dimensión sufrimiento emocional y espiritual, es decir, a mayor edad menor carga de enfermedad. Este hallazgo puede explicarse a partir de teorías sobre la experiencia, regulación y percepción en el proceso de envejecimiento, puesto que se ha reportado un patrón positivo relacionado con la edad, en la respuesta ante situaciones negativas. De esta manera, la percepción de tiempo limitado que tienen las personas mayores los lleva a ser selectivos emocionalmente y a concentrarse en el presente, desarrollando más habilidades de regulación emocional ${ }^{(22)}$.

El tiempo de enfermedad reportó una correlación negativa, débil y significativa con la carga total y todas sus dimensiones, es decir, a mayor tiempo de enfermedad, menor carga. Según otros estudios ${ }^{(23,24)}$, la persona con EC crea una familiaridad con las situaciones derivadas de la enfermedad, adopta nuevos cambios e incorpora estas a su nuevo estilo de vida, dando lugar a que aquello que en un primer momento se interpretó como un problema, con el paso del tiempo se admite como normal.

Por su parte, el número de horas diarias que la persona requiere para su cuidado presentó una correlación positiva, moderada y significativa en la carga total y las dimensiones Disconfort físico y Alteración socio-familiar y cultural, además de una correlación positiva, débil y significativa con la dimensión Sufrimiento emocional y espiritual. 
Según diversos autores ${ }^{(25}$, 26), el requerir cuidado por un gran número de horas al día impacta en la dimensión psicológica y emocional de la persona con EC, pues el perder la independencia y la autonomía en la ejecución de las actividades básicas de la vida diaria genera en el paciente sentimientos de inutilidad, frustración, rechazo y enojo, entre otros, que pueden influir en la percepción de carga que se tiene. Además, también hay afectación en el contexto familiar y en la apreciación del paciente de sentirse como una carga, como consecuencia de las altas demandas de cuidados directos que ocasionan cambios en las dinámicas o roles familiares ${ }^{(27)}$.

De las implicaciones prácticas de este estudio se deriva la importancia de realizar una valoración holística de lo que al paciente le genera carga de su enfermedad. Según los resultados encontrados en este estudio, podría ser útil profundizar respecto del cuidado del paciente referido a los aspectos relacionados con la carga física de la enfermedad, ya que para este, son los de más fácil exploración e identificación. Así mismo, es importante la identificación de lo que genera carga en las dimensiones emocionales y socio-familiares del paciente, puesto que para el caso de las personas con EC, la familia y los aspectos psicológicos y de estado de ánimo son cruciales en el mantenimiento del tratamiento y el afrontamiento de la situación.

Una limitación de este estudio fue el muestreo por conveniencia aun cuando para minimizar sus efectos se trató de incluir participantes de varias instituciones y asistir a los diferentes servicios donde se encontraban las personas con EC. En

\section{REFERENCIAS}

1. OMS. Plan de acción para la prevención y el control de las enfermedades no transmisibles en las Américas 2013-2020 [Internet]. Ginebra; 2013 (citado 23 dic 2018). 64 p. Disponible en: https:// www.paho.org/hq/dmdocuments/2015/planaccion-prevencion-control-ent-americas.pdf

2. Vos T, Abajobir AA, Abate KH, Abbafati C, Abbas KM, Abd-Allah F, et al. Global, regional, and national incidence, prevalence, and years lived with disability for 328 diseases and injuries for 195 countries, 1990-2016: a systematic analysis for the Global Burden of Disease Study 2016. Lancet. 2017; 390(10100): 1211-59. futuras investigaciones conviene conducir estudios con muestreo aleatorio y con poblaciones con EC específicas, ya que, como lo evidencia la literatura, la carga que genera la enfermedad puede llegar a ser distinta de acuerdo al tipo de población estudiada.

\section{CONCLUSIONES}

La Percepción de carga de la enfermedad en personas con EC mostró que, para las personas en este estudio, existe un mayor impacto en la dimensión Disconfort físico que en las dimensiones de Sufrimiento emocional y espiritual o Alteraciones socio-familiar y culturales. Así mismo, pacientes con mayor edad y más tiempo con la EC presentaron menores niveles de Percepción de carga de la enfermedad y aquellos con más necesidades de cuidado, representadas en el número de horas de cuidado diario, presentaron mayores niveles de Percepción de carga de la enfermedad.

Los resultados de este estudio muestran la importancia de una identificación adecuada de la carga que representa la EC para las personas que la padecen, partiendo desde los aspectos más tangibles e instrumentales de los cuidados como el disconfort físico, hasta aquellos de orden emocional y espiritual.

En esta línea de investigación resulta importante conocer si las intervenciones de enfermería existentes pueden abordar y hacer frente, de forma holística, a los diversos aspectos que genera la carga de la EC para paciente.

3. Carreño SP, Sánchez-Herrera B, Carrillo GM, Chaparro-Díaz L, Gómez OJ. Carga de la enfermedad crónica para los sujetos implicados en el cuidado. Rev Fac Nac de Salud Pública. 2016; 34: 342-9.

4. Sassi F. Calculating QALYs, comparing QALY and DALY calculations. Health Policy Plan. 2006; 21(5): 402-8.

5. Sav A, Salehi A, Mair FS, McMillan SS. Measuring the burden of treatment for chronic disease: implications of a scoping review of the literature. BMC Med Res Methodol. 2017; 17(1): 140.

6. Sav A, King MA, Whitty JA, Kendall E, Mcmillan SS, Kelly F, et al. Burden of treatment for chronic illness: A concept analysis and review of the 
literature. Health Expect. Jun 2015; 18(3): 312-24.

7. Sánchez-Herrera B, Carrillo GM, Cárdenas DC, Alarcón AA. Diseño, validez facial y de contenido del instrumento carga de la enfermedad crónica para el paciente - GPCP-UN. Rev Medica Risaralda. 2017; 23(1): 17-21.

8. Carrillo GM, Bayona HA, Arias EM. Perfil y carga de la enfermedad de personas con cáncer gástrico sometidas a gastrectomía. Rev Fac Med. 2018; 66(1): 13-8.

9. Barrera OL, Vargas RE, Cendales PA. Encuesta de caracterización para el cuidado de una persona con enfermedad crónica. Investig Enferm Imagen Desarr. 2015; 17(1): 27-43.

10. Dodd M, Janson S, Facione N, Faucett J, Froelicher ES, Humphreys J, et al. Advancing the science of symptom management. J Adv Nurs. 2001; 33(5): 668-76.

11. Lenz ER, Pugh LC, Milligan RA, Gift A, Suppe F. The Middle-Range Theory of Unpleasant Symptoms: An Update. Adv Nurs Sci. 1997; 19(3): 14-27.

12. Palma A, del Río I, Bonati P, Tupper L, Villarroel L, Olivares P, et al. Frecuencia y pesquisa de síntomas en pacientes crónicos en fases avanzadas en un hospital clínico: ¿Existe concordancia entre pacientes y médicos? Rev Med Chile. 2008; 136(5): 561-9.

13. Goldmann E, Roberts ET, Parikh NS, BodenAlbala B. Chronic physical illness burden and suicidal ideation among dominicans in New York city. J Immigr Minor Health. Jun 2017; 19(3): 616-22.

14. Portz JD, Kutner JS, Blatchford PJ, Ritchie CS. High symptom burden and low functional status in the setting of multimorbidity. J Am Geriatr Soc. Oct 2017; 65(10): 2285-9.

15. Taylor-Clift A, Hobfoll SE, Gerhart JI, Richardson D, Calvin JE, Powell LH. Posttraumatic stress and depression: potential pathways to disease burden among heart failure patients. Anxiety, Stress Coping. 2016; 29(2): 139-52.

16. Kirmayer LJ. Culture and the metaphoric mediation of pain. Transcult Psychiatry. 2008; 45(2): 318-38.

17. Corwin EJ, Berg JA, Armstrong TS, DeVito A, Lee KA, Meek P, et al. Envisioning the future in symptom science. Nurs Outlook. 2014; 62(5): 346-51.

18. Alpert CM, Smith MA, Hummel SL, Hummel EK. Symptom burden in heart failure: assessment, impact on outcomes, and management. Heart Fail Rev [Internet]. 2017 (citado 26 dic 2018); 22(1): 25-39. Disponible en: https://doi.org/10.1007/ s10741-016-9581-4

19. Hwang K-H, Cho O-H, Yoo Y-S. Symptom clusters of ovarian cancer patients undergoing chemotherapy, and their emotional status and quality of life. Eur J Oncol Nurs. 2016; 21:215-22.

20. Breivik H, Collett B, Ventafridda V, Cohen R, Gallacher D. Survey of chronic pain in Europe: prevalence, impact on daily life, and treatment. Eur J Pain. May 2006; 10(4): 287-333.

21. Koné PA, Bronskill SE, Gruneir A, Calzavara A, Thavorn K, Petrosyan Y, et al. The increasing burden and complexity of multimorbidity. BMC Public Health. 2015; 15(1): 415.

22. Isaacowitz DM, Livingstone KM, Castro VL. Aging and emotions: experience, regulation, and perception. Curr Opin Psychol. 2017; 17: 79-83.

23. Penrod J, Hupcey JE, Shipley PZ, Loeb SJ, Baney B. A model of caregiving through the end of life: seeking normal. West J Nurs Res. 2012; 34(2): 174-93.

24. Corbin JM. The Corbin and Strauss Chronic Illness Trajectory model: an update. Sch Inq Nurs Pract. 1998; 12(1): 33-41.

25. Rajala K, Lehto JT, Sutinen E, Kautiainen H, Myllarniemi M, Saarto T. Marked deterioration in the quality of life of patients with idiopathic pulmonary fibrosis during the last two years of life. BMC Pulm Med. 2018; 18(1): 172.

26. Hsu T, Loscalzo $M$, Ramani R, Forman $S$, Popplewell L, Clark K, et al. Are disagreements in caregiver and patient assessment of patient health associated with increased caregiver burden in caregivers of older adults with cancer? Oncologist. 2017; 22(11): 1383-91.

27. Foley G, Timonen V, Hardiman O. "I hate being a burden": The patient perspective on carer burden in amyotrophic lateral sclerosis. Amyotroph Lateral Scler Frontotemporal Degener. 2016; 17(5-6): 351-7. 